\title{
Deep Brain Stimulation Rescues Memory and Synaptic Activity in a Rat Model of Global Ischemia
}

\author{
Elise Gondard, ${ }^{1}$ Lucy Teves, ${ }^{1 *}$ Lihua Wang, ${ }^{1 *}$ (C) Chris McKinnon, ${ }^{1}$ Clement Hamani, ${ }^{2,3,4}$ CSuneil K. Kalia, ${ }^{1,4}$ \\ (D) Peter L. Carlen , 1,4 (D) Michael Tymianski, ${ }^{1,4}$ and (D)Andres M. Lozano ${ }^{1,4}$ \\ ${ }^{1}$ Krembil Research Institute, Toronto Western Hospital, University Health Network, Toronto, Ontario M5T 2S8, Canada, ${ }^{2}$ Research Imaging Centre, \\ ${ }^{3}$ Campbell Family Mental Health Research Institute, Centre for Addiction and Mental Health, Toronto, Ontario M5T 1R8, Canada, and ${ }^{4}$ Division of \\ Neurosurgery, Department of Surgery, Toronto Western Hospital, University of Toronto, Toronto, Ontario M5T 2S8, Canada
}

Ischemic stroke is responsible for a large number of neurological deficits including memory impairment. Deep brain stimulation (DBS), a well established therapeutic modality for the treatment of movement disorders, has recently shown potential beneficial effects on memory in animals and patients with Alzheimer's disease. Here, we test DBS for its ability to improve memory impairments by stimulating the entorhinal cortex (EC) in a rat model of global ischemia (GI). Two weeks after GI, adult male rats received high-frequency EC DBS for $1 \mathrm{~h}$, and animals were assessed for changes in locomotor activity, learning, and memory 6 weeks later. GI produced spatial memory impairment that was ameliorated by DBS, with no difference between the group that received DBS for GI (GI-DBS ON group) and nonstroke control groups. Although GI led to a dramatic CA1 neuronal loss that could not be rescued with DBS, stimulation attenuated the reduction of CA1 synaptophysin expression after GI. Further, in vitro slice recordings showed a restoration of typical evoked synaptic dendritic fields in GI-DBS ON animals, indicating that the DBS-induced memory rescue is associated with increased synaptophysin expression and enhanced synaptic function. These results suggest that DBS may ameliorate the functional consequences of cerebral ischemia and point to be a potential new therapeutic approach.

Key words: deep brain stimulation; global ischemia; memory; Morris water maze; synaptic activity; synaptophysin

\section{Significance Statement}

Deep brain stimulation (DBS) is remarkably effective in treating Parkinson's disease and is currently under investigation for the treatment of neuropsychiatric disorders including Alzheimer's disease. Until now, DBS has not been examined for its cognitive benefits in the context of hypoxic-ischemic injuries. Here, we investigated the effect of DBS in a rat model of global ischemia (GI) that mimics the neurological consequences occurring after a cardiac arrest. We show that DBS rescues memory deficits induced by GI and produces changes in synaptic activity in the hippocampus. Novel approaches to improve neurological outcomes after stroke are urgently needed; therefore, the present study highlights a possible role for DBS in the treatment of cognitive impairment associated with ischemia.

\section{Introduction}

Stroke is among the most frequent cause of neurological disability and the second leading cause of death in adults worldwide

\section{Received May 14, 2018; revised Jan. 7, 2019; accepted Jan. 11, 2019}

Author contributions: E.G., C.H., M.T., P.L.C., and A.M.L. designed research; E.G., L.T., L.W., and C.M. performed research;S.K.K. contributed unpublished reagents/analytic tools; E.G., L.W., and C.M. analyzed data; E.G. wrote the paper.

This research was supported by Neuron (Network of European Funding for Neuroscience Research) and the Canadian Research Chair Program. We thank Hien Chau, Desirée Marillac, and Jorge Milsted for their assistance in the laboratory and Dr. Eubanks laboratory for helping to configure and prepare the locomotor apparatus.

C.H. received honoraria from Medtronics. The authors declare no other competing financial interests.

*L.T. and L.W. contributed equally to the data and manuscript.

Correspondence should be addressed to Andres M. Lozano at lozano@uhnresearch.ca or Elise Gondard at elisegondard@yahoo.fr.
(Lackland et al., 2014; Mozaffarian et al., 2015). Stroke often produces long-term neurological deficits in various functions including locomotion, speech, and memory (Schweizer and Macdonald, 2014). Cardiac arrest, myocardial infarction, carbon monoxide inhalation, and asphyxia can cause temporary global cerebral ischemia, a stroke subtype (Madl and Holzer, 2004). These hypoxic-ischemic injuries elicit a delayed neuronal death in vulnerable structures including the hippocampus (SchmidtKastner and Freund, 1991; Desmond et al., 2000). The CA1 area of the hippocampus for example has been reported to be the most vulnerable region, causing impaired cognition and memory def- 


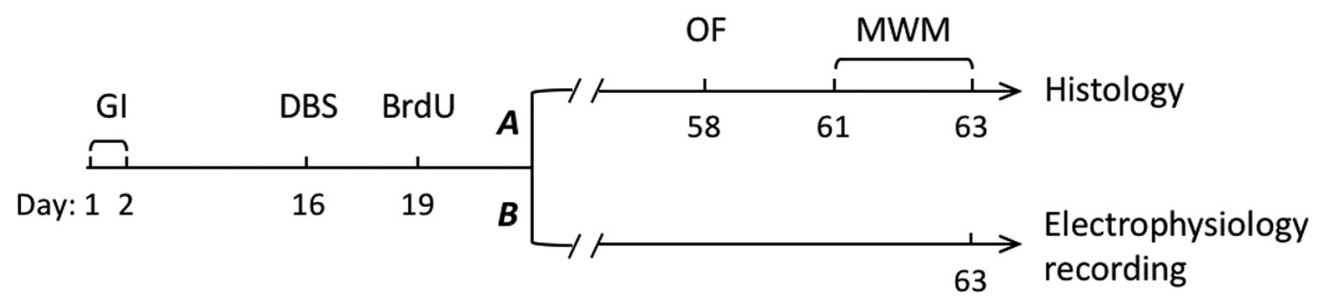

Figure 1. Experimental design. All animal underwent $\mathrm{Gl}$ surgery at day 1 and day 2 . Gl was induced to $\mathrm{Gl}$ rats, whereas NS control animals underwent surgery with no $\mathrm{Gl}$ induction. After 2 weeks of recovery, all rats underwent bilateral implantation of electrodes (DBS) in the EC. DBS ON rats received $1 \mathrm{~h}$ of stimulation, whereas DBS OFF animals received no stimulation. Animals were divided into four different groups: NS-DBS OFF ( $n=21)$; NS-DBS ON $(n=23)$; GI-DBS OFF $(n=22)$; and GI-DBS ON $(n=23)$. Three days after EC stimulation, rats were injected with BrdU ( 4 injections of $50 \mathrm{mg} / \mathrm{kg}$ doses). Animals were then randomly assigned to experiment A or B. Experiment A, At day 58, the locomotion of animals was assessed in the open field (OF), and $6.5 \mathrm{weeks}$ after DBS animals were tested in the MWM, with 3 trials per day during $3 \mathrm{~d}$ where memory performance was assessed during the probe test, $4 \mathrm{~h}$ after the last training trial at day 63 . Animals were killed after the probe test for histology analysis. NS-DBS OFF ( $n=17)$, NS-DBS ON $(n=18)$, GI-DBS OFF $(n=16)$, and GI-DBS ON ( $n=18)$. Experiment B, At day 63, animals were killed to perform slice in vitro recordings in CA1. NS-DBS OFF $(n=4)$, NS-DBS ON $(n=5)$, GI-DBS OFF $(n=6)$, and GI-DBS ON $(n=5)$.

icits within days in survivors of cardiac arrest (Volpe and Petito, 1985; Petito et al., 1987). New approaches are urgently needed to improve patient outcomes after ischemia. A number of novel treatment modalities are being tested in an attempt to drive poststroke neuroplasticity and enhance functional recovery (Azad et al., 2016). Previous preclinical studies have shown that chronic electrical stimulation of the dentatothalamocortical (DTC) pathway can enhance motor recovery (Adkins-Muir and Jones, 2003; Machado et al., 2013; Cooperrider et al., 2014), but the effects of stimulation on ischemia-induced memory deficits remain unexamined.

Deep brain stimulation (DBS) is a well established treatment in a range disorders including Parkinson's disease, essential tremor, dystonia, and is currently being evaluated in pain and psychiatric illness (Lozano and Hamani, 2004; Mayberg et al., 2005; Ponce and Lozano, 2010; Kalia et al., 2013; Lipsman et al., 2013). It is estimated that $>160,000$ patients worldwide have been implanted with DBS systems, with the rate of annual accrual increasing (Lozano and Lipsman, 2013). The potential beneficial effects of DBS have been reported recently for memory enhancement in rodents (Hamani et al., 2011; Stone et al., 2011; Hescham et al., 2013) and humans (Hamani et al., 2008; Laxton et al., 2010; Smith et al., 2012; Lozano et al., 2016). Our previous studies (Laxton et al., 2010; Sankar et al., 2015) showed promising effects of DBS in patients with Alzheimer's disease (AD) with respect to cognitive function, disease progression, and increased cortical glucose metabolism contrary to the expected longitudinal decline generally seen in $\mathrm{AD}$ patients. In naive adult mice, $1 \mathrm{~h}$ of stimulation of the entorhinal cortex (EC) increased spatial memory function by promoting hippocampal neurogenesis. This memory facilitation was seen when mice were trained 6 weeks after stimulation, but not at 1 week (Stone et al., 2011). We also found that chronic EC DBS in an AD mouse model improved memory and cleared AD-related proteins, and increased neurogenesis and the level of synaptophysin in CA1 (Akwa et al., 2018; Mann et al., 2018). Furthermore, acute stimulation of limbic structures increased the expression of neurotrophic factors and synaptic proteins that are known to play a crucial role in memory functions (Toda et al., 2008; Encinas et al., 2011; Gondard et al., 2015; Hao et al., 2015; Akwa et al., 2018).

Based on these findings, we hypothesized that stimulating the neural circuits underlying learning and memory could improve memory impairments observed in a rat model of global ischemia (GI). GI mimics the neurological consequences occurring after a cardiac arrest by inducing delayed neuronal death in the CA1 region, reaching maximal effect within 1-2 weeks dependent on the duration of the ischemic insult (Pulsinelli et al., 1982; Col- bourne et al., 1999). This loss of the CA1 pyramidal neurons is associated with severe impairments of hippocampal-dependent memory, such as spatial learning and memory (Nunn et al., 1994; Block, 1999; Hartman et al., 2005; von Euler et al., 2006). Thus, we investigated here the effect of acute EC DBS on memory and plasticity in a rat model of GI.

\section{Materials and Methods}

A summary of our experimental design and timeline is outlined in Figure 1.

\section{Animals}

This study was approved by the Toronto Western Research Institute Animal Care Committee and was conducted in accordance with the guidelines of the Canadian Council on Animal Care. Adult male Wistar rats $(180-200 \mathrm{~g})$ were housed with ad libitum access to food and water in a room maintained at a constant temperature $\left(20-22^{\circ} \mathrm{C}\right)$ and on a $12 \mathrm{~h}$ light/dark cycle.

\section{Global ischemia}

We used four-vessel occlusion to produce GI (Pulsinelli and Buchan, 1988; Sun et al., 2009). Briefly, at day 1, rats were anesthetized with isoflurane. Body temperature was controlled and maintained at $37 \pm$ $0.5^{\circ} \mathrm{C}$ by a heating lamp. The vertebral arteries on both sides were exposed and permanently occluded by cauterization and animals allowed to awaken. On day 2, rats underwent repeat anesthesia, and both common carotid arteries were exposed and occluded by clipping for $15 \mathrm{~min}$. During carotid artery ligation, the following parameters were assessed to assure the successful occlusion: completely flat bitemporal electroencephalogram, dilated pupils, absence of corneal reflex and steady body temperature readings. Subsequently, ligatures were removed, and the recirculation of blood flow was confirmed by observation. Animals were allowed to wake up and returned to their home cage for 2 weeks of recovery. Control nonstroke (NS) animals underwent surgery but did not have vessel occlusion.

\section{Electrical stimulation of the entorhinal cortex and bromodeoxyuridine administrations}

Two weeks after the GI induction, animals were anesthetized with isoflurane and had their heads fixed in a stereotactic instrument (model 900, David Kopf Instruments). Concentric platinum bipolar electrodes (cathode tip with $0.1 \mathrm{~mm}$ diameter and $0.25 \mathrm{~mm}$ of exposed length; model SNEX-100, Rhodes Medical Instruments) were implanted into the EC bilaterally at the following coordinates relative to bregma: anteroposterior, $-6.5 \mathrm{~mm}$; mediolateral, $5 \mathrm{~mm}$; dorsoventral, $9 \mathrm{~mm}$ (Paxinos and Watson, 2008). Stimulation was applied with a handheld stimulator (model 3628 screener, Medtronic) for $1 \mathrm{~h}$ at parameters that were similar to those in our previous report (DBS ON group: $2.5 \mathrm{~V}, 90 \mu$ s of pulse width, 130 Hz frequency; Toda et al., 2008; Hamani et al., 2011; Gondard et al., 2015). Following stimulation, electrodes were removed, the surgical incision was closed, and the animals were allowed to recover. Control animals had electrodes implanted but did not receive stimulation (DBS OFF group). 
Animals received the thymidine analog bromodeoxyuridine (BrdU; Sigma-Aldrich) injections $3 \mathrm{~d}$ after DBS surgery to assess neurogenesis. The BrdU was dissolved in sterile saline and heated to $50-60^{\circ} \mathrm{C}$ at a concentration of $25 \mathrm{mg} / \mathrm{ml}$. Similarly to our previous report (Toda et al., 2008), animals received a total of $200 \mathrm{mg} / \mathrm{kg} \mathrm{BrdU}$ (50 mg/kg, i.p., with four injections every $3 \mathrm{~h}$ ).

\section{Animal groups and experimental procedures}

A total of 97 rats underwent the surgical procedure, as follows: 53 rats received GI, and 44 were assigned to the NS control group. After histology analysis, 8 of the 53 GI rats were excluded due to incomplete global ischemia. Animals were divided into the following two subgroups for DBS stimulation: from the GI groups, there were 23 animals in the DBS-ON group and 22 animals in the DBS-OFF group, with the electrodes implanted with no stimulation. From the NS control group, 23 animals were stimulated (DBS ON) and 21 animals received the implant without simulation (DBS-OFF).

Behavioral and histological evaluations were assessed in Experiment $\mathrm{A}$ ( $n=69$ ); electrophysiology recordings were performed in Experiment $\mathrm{B}$ ( $n=20$; described below). Behavioral and electrophysiology experiments were performed 6 weeks after DBS, based on our previous findings showing $1 \mathrm{~h}$ of EC-DBS enhanced spatial memory performance when mice were trained 6 weeks after stimulation (Stone et al., 2011). The following animals were used in experiment A: NS-DBS OFF $(n=17)$, NS-DBS ON $(n=18)$, GI-DBS OFF $(n=16)$, and GI-DBS ON $(n=18)$. The following animals were used in experiment B: NS-DBS OFF $(n=4)$, NS-DBS ON $(n=5)$, GI-DBS OFF $(n=6)$, and GI-DBS ON $(n=5)$.

\section{Experiment $A$}

Locomotor activity measurement in the open field. Five and one-half weeks after BrdU injections, animals were tested in an open field to assess their locomotor activity. An automated movement detection system (AM1053 activity monitors, Linton Instrumentation) was used to measure motor behavior in the open-field arena. Briefly, this apparatus consists of a Plexiglas box surrounded by a housing frame $(45 \times 25 \mathrm{~cm})$ that houses an array of 24 infrared beams forming a grid across two levels. As the rat moves, a beam is broken and an activity count, dependent on the type of movement, is registered. Several behavioral parameters, including travel distance and the of total number of rearing events, were measured using this system. These behavioral assessments were conducted between 10:00 A.M. and 1:00 P.M. to minimize circadian effects, and each subject was examined in the open field only once, for $60 \mathrm{~min}$, to prevent acclimation to the open-field apparatus.

Morris water maze apparatus and procedures. The Morris water maze (MWM) procedures have been described previously (Stone et al., 2011; Mann et al., 2018). A circular water-maze tank $(200 \mathrm{~cm}$ diameter, $75 \mathrm{~cm}$ high), constructed of dark blue plastic was located in a dimly lit room. The water was maintained at $23 \pm 2{ }^{\circ} \mathrm{C}$ and was made opaque by the addition of a blue nontoxic paint. A square escape platform $(12 \times 12 \mathrm{~cm})$ was submerged $1.5 \mathrm{~cm}$ below the water surface, in a fixed position in one quadrant. White curtains with distinct cues painted on them surrounded the pool. A video camera was mounted in the ceiling above the pool and was connected to a video recorder and tracking device (SMART tracking software), which permitted on-line and off-line automated tracking of the paths taken by the rats. Before training, rats were individually handled for 2 min each day over 7 consecutive days. Rats were trained over 3 consecutive days with three trials per day (intertrial interval, $5 \mathrm{~min}$ ). On each trial, rats were placed into the pool, facing the wall, in one of three start locations (the order of which was pseudorandomly varied throughout training). The trial was complete once the rat found the platform and remained on the platform for 10 or $60 \mathrm{~s}$. If the rat failed to find the platform in this time frame, the experimenter gently guided the rat onto the platform. After the completion of training, spatial memory was assessed during a probe trial $4 \mathrm{~h}$ after the final session on day 3 . Behavioral data from training trials and probe tests were acquired and analyzed using an automated tracking system (SMART).

Histology and immunohistochemistry. Ninety minutes after the completion of the probe test, animals were deeply anesthetized and transcardially perfused with normal saline, followed by a $4 \%$ paraformaldehyde
(PFA) solution. Brains were removed, postfixed overnight in PFA, transferred into $30 \%$ sucrose for $3-4 \mathrm{~d}$ at $4^{\circ} \mathrm{C}$ and then stored at $-80^{\circ} \mathrm{C}$. Free-floating coronal $40 \mu \mathrm{m}$ sections were cut on a cryostat, collected in seven parallel sets (each slice $280 \mu \mathrm{m}$ apart from the next), placed in cryoprotectant, and processed for immunohistochemistry.

The following primary antibodies were used: mouse monoclonal antiNeuN (1:1000; Millipore Bioscience Research Reagents), rat monoclonal anti-BrdU for BrdU (1:200; Bio-Rad), and mouse monoclonal antisynaptophysin (1:500; Sigma-Aldrich).

Diaminobenzene immunohistochemistry. NeuN and synaptophysin immunoreactivities were examined using diaminobenzene (DAB) immunohistochemistry. Sections were pretreated with $0.25 \%$ Triton X-100 for $30 \mathrm{~min}$ followed by $5 \%$ normal goat serum for $30 \mathrm{~min}$. Sections were then incubated with primary antibodies overnight at $4^{\circ} \mathrm{C}$. After $2 \mathrm{~h}$ of incubation with a secondary antibody (1:200; goat biotin-SP anti-mouse IgG, Jackson ImmunoResearch) at room temperature, sections were treated with avidin-biotin complex (Vectastain Elite ABC Kit, Vector Laboratories) for $1 \mathrm{~h}$ and visualized with a DAB reaction (Vector Laboratories).

Fluorescence immunohistochemistry. Detailed $\mathrm{BrdU}^{+} / \mathrm{NeuN}^{+}$staining procedures have been described previously (Stone et al., 2011; Mann et al., 2018). In brief, sections were pretreated with $2 \mathrm{~N} \mathrm{HCl}$ at $37^{\circ} \mathrm{C}$ for 30 $\mathrm{min}$, neutralized in $0.1 \mathrm{~m}$ borate, and blocked in 3\% normal goat serum for $1 \mathrm{~h}$. Sections were then incubated with primary rat anti-BrdU and $\mathrm{NeuN}$ antibodies overnight at $4^{\circ} \mathrm{C}$. Sections were washed and incubated during $2 \mathrm{~h}$ with secondary fluorescent-conjugated antibodies: Alexa Fluor-488 anti-rat (1:500; Invitrogen), Alexa Fluor-594 anti-mouse (1: 500; Invitrogen). Sections were slide mounted with Dako Permafluor Anti-Fade Medium (Agilent).

Imaging and quantification. Image analysis was performed by an experimenter blinded to the treatment condition. DAB-stained slides were scanned with ScanScope XT scanner at $20 \times$ magnification. Global ischemia-related damage to the CA1 region was visualized by Cresyl Violet and NeuN staining. HALO Image Analysis Software (Indica Labs) was used to quantify the total number of $\mathrm{NeuN}^{+}$cells in the granule cell layers of CA1, CA3, and dentate gyrus (DG) in every seventh section, with a total of six randomly selected rats studied per group.

Densitometric analysis of synaptophysin immunoreactivity was performed using Aperio ImageScope, at $20 \times$ magnification. For CA1, CA3, and DG, four measurements were taken from each region per hemisphere, plus a background measurement using an identical box size. An average optical density measurement was calculated from a total of four sections per rat, with background correction. A total of eight randomly selected rats were studied per group.

Immunofluorescence-stained sections were scanned with a 3DHistech Pannoramic 250 Flash II Slide Scanner at $20 \times$ magnification. BrdU ${ }^{+} /$ $\mathrm{NeuN}^{+}$cell counting was limited to the DG granule cell layer and the region comprising the $40 \mu \mathrm{m}$ border along the hilar margin (subgranular zone). $\mathrm{BrdU}^{+} / \mathrm{NeuN}^{+}$colabeling was measured in every seventh section (that is, $280 \mu \mathrm{m}$ apart) throughout the rostrocaudal segment of the granule cell layer. All $\mathrm{BrdU}^{+} / \mathrm{NeuN}^{+}$cells were counted bilaterally in each of the sections. The resulting cell numbers were multiplied by 7 (the number of sets of slices per animal) to obtain the estimated total number of positive cells per DG. After identification, apparent double-labeled cells were confirmed by confocal microscopy. The proportions of BrdU ${ }^{+}$cells colabeled with NeuN in the DG was calculated for eight randomly selected rats per group.

\section{Experiment B: electrophysiology recordings}

Six and one-half weeks after DBS, rats were anesthetized with pentobarbital $(75 \mathrm{mg} / \mathrm{kg})$ and decapitated (Fig. 1B). Each brain was quickly removed, and coronal slices containing the hippocampus, $500 \mu \mathrm{m}$ thick, were prepared using a vibratome (model 1200VT, Leica) using an icecold oxygenated $\left(95 \% \mathrm{O}_{2} / 5 \% \mathrm{CO}_{2}\right)$, aCSF cutting solution containing sucrose [composition as follows (in $\mathrm{mM}$ ): sucrose (248), $\mathrm{KCl}(2)$, $\mathrm{MgSO}_{4}(3), \mathrm{CaCl} 2$ (1), $\mathrm{NaHCO} 3$ (26), $\mathrm{NaH}_{2} \mathrm{PO}_{4}$ (1.25), D-glucose (10)]. Slices were transferred to a chamber with recording aCSF [composition as follows (in $\mathrm{mm}$ ): $\mathrm{NaCl}$ (123), $\mathrm{KCl}$ (3.5), $\mathrm{CaCl} 2$ (1.5), $\mathrm{MgSO}_{4}(2)$, $\mathrm{NaHCO} 3$ (25), $\mathrm{NaH}_{2} \mathrm{PO}_{4}$ (1.2), and D-glucose (10), equilibrated with 
A

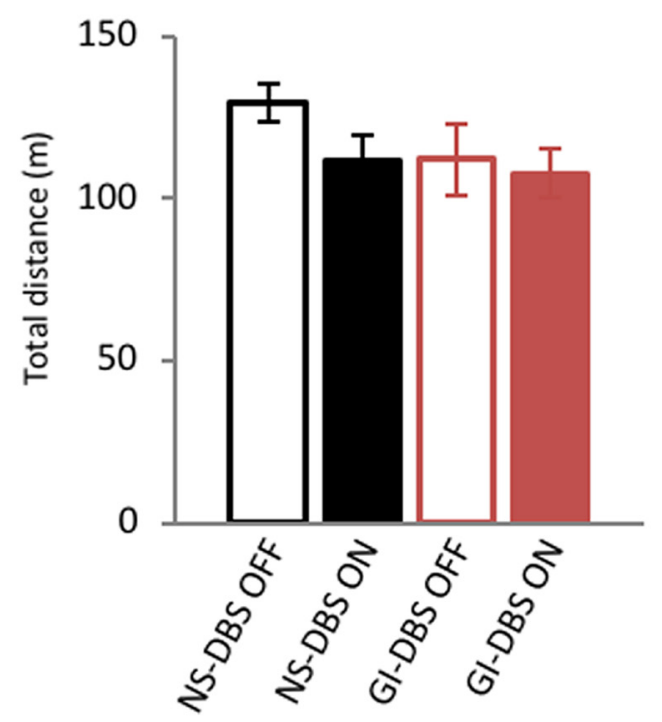

B

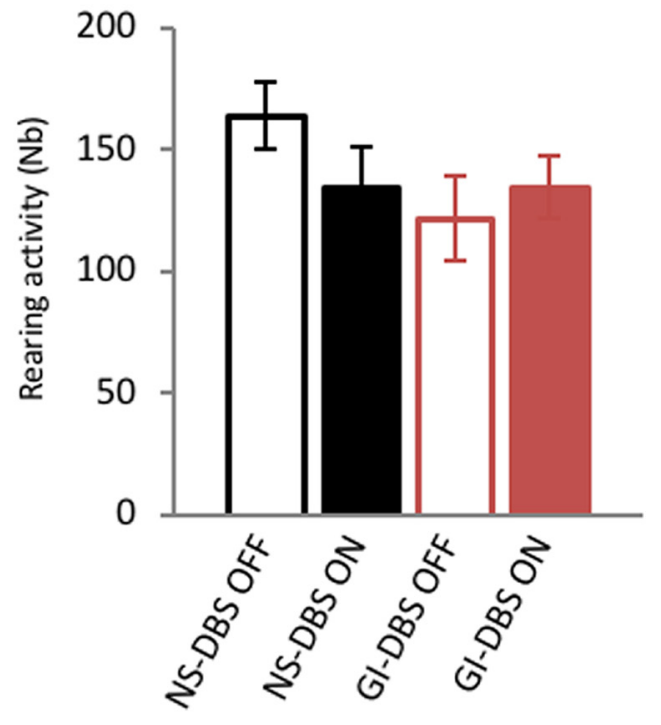

Figure 2. Gl and/or DBS treatments did not affect locomotor activity in the OF. $\boldsymbol{A}$, Total distance traveled. $\boldsymbol{B}$, Rearing activity. There were no differences among the four groups in the total distance traveled and the rearing activity. NS-DBS OFF, $n=17$; NS-DBS ON, $n=18 ; \mathrm{GI}-\mathrm{DBS}$ OFF, $n=16$; and GI-DBS ON, $n=18$. Data are presented as the mean \pm SEM.

carbogen at $32^{\circ} \mathrm{C}$ for $30 \mathrm{~min}$, and then room temperature for $60 \mathrm{~min}$ before experiments].

Recordings were performed in a submerged recording chamber continuously perfused with oxygenated recording aCSF at a flow rate of 6 $\mathrm{ml} / \mathrm{min}$ at $32-34^{\circ} \mathrm{C}$. Field potentials were recorded extracellularly through glass pipettes filled with aCSF freshly made on the day of experiment. The electrode (tip 4-5 $\mu \mathrm{m}$ ) was placed in the CA1 region either in the somatic or dendritic (den) layer. Electrical orthodromic stimulation of Schaffer collateral afferents was performed using a bipolar tungsten electrode. Current pulses of 100 ms duration were generated through a stimulator (Grass Technologies) and delivered through an isolation unit every $20 \mathrm{~s}$. An input-output relationship was first established when threshold and maximal responses were identified as $0 \%$ and $100 \%$, respectively. Evoked responses were recorded by increasing the stimulation intensity in $10 \%$ steps. The stimulation strength was used when the response was at $\sim 90 \%$ maximum when the amplitudes of evoked responses were measured. Maximum transmissions were used when slopes were compared between groups.

All recordings were made using a Multiclamp 700B Amplifier and stored in a DigiData 1322 Acquisition System (Molecular Devices). Data were collected only after stable field potentials were recorded, and averages were obtained from four recordings. All data were analyzed with Pclamp software (version 10.3, Axon Instruments).

\section{Data analysis and statistics}

Experimental data followed a normal distribution, as assessed by the d'Agostino-Pearson omnibus normality test. Behavioral, immunohistochemistry, and electrophysiology data are presented as the mean \pm SEM and were evaluated using two-way ANOVAs with statistical significance set at $p \leq 0.05$, followed by a Tukey's post hoc correction for multiple comparisons where applicable (GraphPad Prism, GraphPad Software). Statistical analysis for the open-field, water maze probe test and cell counting was performed using two-way ANOVA. For water maze training sessions, multivariate ANOVA with one level repeated (day of testing) and one level not repeated (treatment) was used to analyze the acquisition data over the days of training.

\section{Results}

GI and DBS treatments did not affect locomotion

Animals with global ischemia preserved their locomotor activity. Neither global ischemia nor DBS stimulation had an effect on locomotor performance measured 8 weeks after ischemia. No significant differences were observed in the total distance traveled (two-way ANOVA: GI treatment, $F_{(1,65)}=1.727, p=0.1934$; DBS treatment, $F_{(1,65)}=1.744, p=0.1913$; interaction, $F_{(1,65)}=$ $0.7585, p=0.3870$; Fig. $2 B$ ) and the rearing activity (two-way ANOVA: GI treatment, $F_{(1,65)}=1.783, p=0.1865$; DBS treatment, $F_{(1,65)}=0.3145, p=0.5769$; interaction, $F_{(1,65)}=1.954$, $p=0.167$; Fig. $2 B)$.

\section{EC stimulation normalized Morris water maze memory deficits in a GI model}

To determine whether EC stimulation could improve memory impairments induced by GI, all rats were trained in an MWM for $3 \mathrm{~d}$ followed by a probe test (Sun et al., 2009; Stone et al., 2011; Mann et al., 2018). There were no significant main effects of treatment on the escape latencies among the four groups during acquisition training, because all groups learned to locate the platform after $3 \mathrm{~d}$ (two-way repeated-measures ANOVA: day, $F_{(2,130)}=114.7, p<0.0001$; Fig. $3 A$ ). During the third training day, however, GI-DBS OFF rats took more time to reach the platform than NS-DBS ON animals $(30.3 \pm 3.5$ vs $16.9 \pm 2 \mathrm{~s}$; Tukey's post hoc test, $p=0.012$ ). Spatial memory was then assessed in a probe test $4 \mathrm{~h}$ later (Fig. $3 B, C$ ). The platform was removed from the pool, and the time rats spent in a quadrant target zone centered on the platform location during training and the number of crossings of the platform zone were measured. Two-way ANOVA revealed significant main effects among the four groups for time spent in the quadrant (GI treatment, $F_{(1,65)}=12.82, p=0.0007$; DBS treatment, $F_{(1,65)}=3.899, p=$ 0.053; Fig. $3 B$ ) and in the number of the platform area crossings (GI treatment, $F_{(1,65)}=4.591, p=0.036$; DBS treatment, $F_{(1,65)}=4.822, p=0.032$; Fig. $\left.3 C\right)$. GI-DBS OFF animals spent less time in the region of the pool that formerly contained the platform compared with NS-DBS OFF ( $7 \pm 0.6$ vs $11.7 \pm 1 \mathrm{~s}, p=$ $0.002)$ and NS-DBS ON $(7 \pm 0.6$ vs $11.8 \pm 0.9 \mathrm{~s}, p=0.001)$. Moreover, GI-DBS OFF crossed the platform zone significantly less often (1.5 \pm 0.2 times) compared with NS-DBS OFF (2.7 \pm 0.5 times, $p=0.041)$ and NS-DBS ON $(2.8 \pm 0.3$ times, $p=$ 0.017 ), suggesting that GI induced spatial memory impairment. Interestingly, GI-DBS ON rats showed an improvement com- 
A

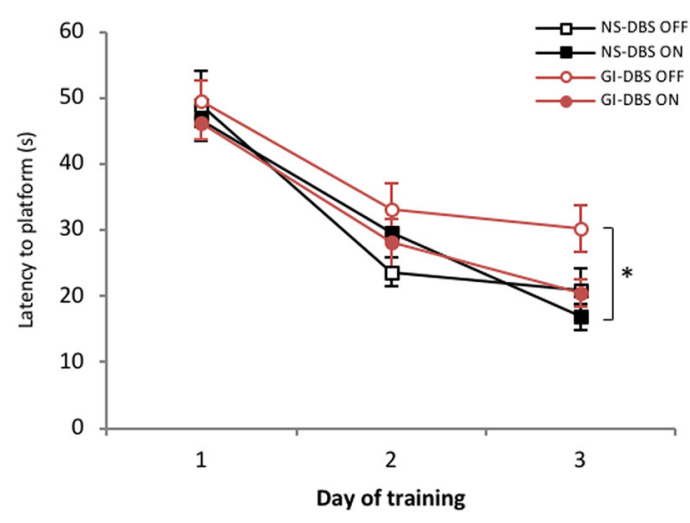

B

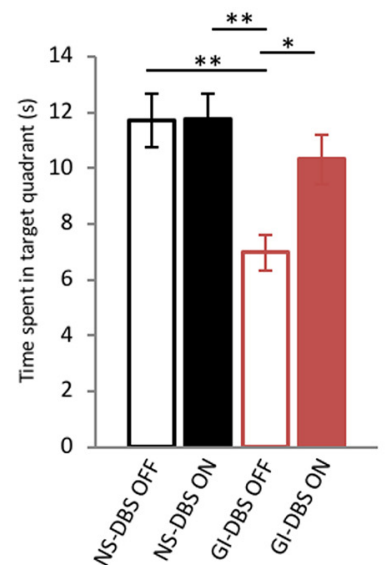

C Probe

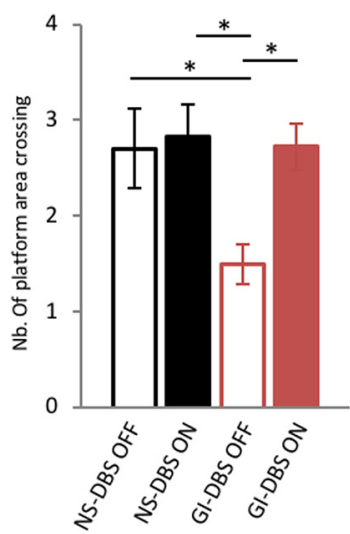

Figure 3. ECDBS in GI rats rescues spatial memory performance to sham in the $4 \mathrm{~h}$ MWM probe test. $A$, All groups learned to locate the platform after $3 \mathrm{~d}$. During the third day of training, GI-DBS OFF rats took more time to reach the platform than NS-DBS ON rats. $B, C$, Memory performance was assessed in a probe test. $B, G$ G-DBS OFF rats spent less time in the region of the pool that formerly contained the platform compared with NS-DBS OFF and NS-DBS ON rats. GI-DBS ON rats spent significantly more time in the platform area than GI-DBS OFF rats. C, GI-CTL rats showed fewer platform area crossings than NS-DBS OFF, NS-DBS ON, and GI-DBS ON rats. Two-way ANOVA followed by Tukey's post hoc test: ${ }^{*} p=0.05,{ }^{* *} p=0.01$. NS-DBS OFF, $n=17 ;$ NS-DBS ON, $n=18 ; \mathrm{GI}-\mathrm{DBS}$ OFF, $n=16$; and GI-DBS ON, $n=18$. Data are presented as the mean \pm SEM.

pared with GI-DBS OFF rats and normalized function back to NS control rats. In GI rats, not only did DBS significantly increase the time spent in the target zone to GI-DBS OFF rats $(10.3 \pm 0.9$ vs $7 \pm 0.6 \mathrm{~s}$; Tukey's post hoc test, $p=0.041$ ), but DBS also improved performance to a level similar to that of NS animals with or without stimulation $(p>0.05)$. Furthermore, the GI-DBS ON group also had a significantly higher number of platform zone crossings compared with GI-DBS OFF rats $(2.7 \pm 0.2$ vs $1.5 \pm$ $0.2, p=0.034)$, but was not significantly different from NS control groups $(p>0.05)$. These results indicate that EC stimulation can rescue spatial memory impairment induced by GI.

\section{Degeneration of CA1 neurons after global ischemia}

Global ischemia rats showed marked reductions of Cresyl Violetstained cells in the CA1 in GI rats compared with NS (Fig. 4A). Staining for the specific neuronal nuclei marker NeuN confirmed the large loss of CA1 neurons in the ischemia group (two-way ANOVA: GI treatment, $F_{(1,20)}=177.5, p<0.0001$; DBS treatment, $F_{(1,20)}=0.775, p=0.389$; interaction, $F_{(1,20)}=0.006, p=$ 0.939; Fig. $4 A, B)$. Quantification of the number of $\mathrm{NeuN}^{+}$neurons in CA1 showed an $\sim 85 \%$ decrease in both the GI-DBS OFF and GI-DBS ON groups ( $12 \pm 5.2$ and $18.2 \pm 6.7 \%$, respectively) compared with the NS-DBS OFF and NS-DBS ON groups ( $100 \pm$ 8.2 and $105 \pm 5.8 \%$, respectively; $p<0.0001)$. No change was found in the number of neurons in the CA3 (two-way ANOVA: GI treatment, $F_{(1,20)}=1.288, p=0.27$; DBS treatment, $F_{(1,20)}=$ $0.353, p=0.559$; interaction, $F_{(1,20)}=0.208, p=0.653$; Fig. $\left.4 B\right)$ and dentate gyrus (two-way ANOVA: GI treatment, $F_{(1,20)}=$ 4.088, $p=0.06$; DBS treatment, $F_{(1,20)}=0.598, p=0.45$; interaction, $F_{(1,20)}=2.155, p=0.158$; Fig. $\left.4 B\right)$ in response to ischemia and/or DBS treatment.

\section{GI-reduced hippocampal neurogenesis}

We previously found that $1 \mathrm{~h}$ of the EC stimulation facilitates MWM memory in adult mice by promoting adult neurogenesis in the DG (Stone et al., 2011). Therefore, we examined whether the improvement in spatial memory induced by EC stimulation in GI rats could be neurogenesis dependent by measuring the number of $\mathrm{BrdU}^{+}$cells expressing NeuN in the DG (Fig. 5).
Two-way ANOVA indicated a significant main effect on the number of $\mathrm{BrdU}^{+} / \mathrm{NeuN}^{+}$cells (two-way ANOVA: GI treatment, $F_{(1,28)}=125.8, p<0.0001$; DBS treatment, $F_{(1,28)}=8.502$, $p=0.007$; interaction, $\left.F_{(1,28)}=6.045, p=0.02\right)$. GI induced a large decrease in hippocampal neurogenesis. GI-DBS OFF rats had significantly fewer $\mathrm{BrdU}^{+} / \mathrm{NeuN}^{+}$cells in the DG than NSDBS OFF rats (202.9 \pm 22 vs $669.8 \pm 75.2$; Tukey's post hoc test, $p<0.0001)$. Although EC DBS significantly increased the number of BrdU ${ }^{+} / \mathrm{NeuN}^{+}$cells in NS rats (NS-DBS ON, 956.3 \pm 67.1 vs NS-DBS OFF, $669.8 \pm 75.2 ; p=0.004)$, EC stimulation was ineffective in increasing neurogenesis in animals with GI $\left(202.9 \pm 22\right.$ vs $227.3 \pm 26.9 ; \mathrm{BrdU}^{+} / \mathrm{NeuN}^{+}$cells, DBS ON vs DBS OFF, $p=0.99$ ). These results suggest that the memory rescue observed in the GI-DBS ON animals was not neurogenesis dependent.

\section{DBS attenuated the decrease in synaptophysin in CA1 observed after ischemia}

We tested whether the positive behavioral outcomes seen in GIDBS ON rats could in part be explained by increased synaptic activity in the hippocampus. Because synaptophysin is thought to be in virtually every synapse, we examined the pattern of synaptophysin immunoreactivity in hippocampal sections. Two-way ANOVA revealed significant main effects of synaptophysin expression in CA1 among the four groups (GI treatment, $F_{(1,28)}=$ 14.02, $p=0.0008$; DBS treatment, $F_{(1,28)}=4.162, p=0.049$; Fig. $6)$. We found a significant decrease in synaptophysin expression in CA1 in the GI-DBS OFF group compared with the NS-DBS OFF group $(-8.8 \pm 1.3 \%, p=0.032)$ and NS-DBS ON group $(-12.3 \pm 2 \%, p=0.002)$. Consistent with the notion that DBS restores synaptic loss after ischemia, we found that no significant differences were observed between GI-DBS ON and NS-DBS OFF $(-3.6 \pm 0.5 \%, p=0.634)$, NS-DBS ON $(-7.1 \pm 1.2 \%, p=$ $0.107)$, and GI-DBS OFF $(+5.2 \pm 0.8 \%, p=0.326)$ groups. Synaptophysin expression remained unchanged in CA3 (two-way ANOVA: GI treatment, $F_{(1,28)}=1.233, p=0.276$; DBS treatment, $F_{(1,28)}=0.071, p=0.792$; interaction, $F_{(1,28)}=2.482, p=0.126$; Fig. $6 B)$ and dentate gyrus (two-way ANOVA: GI treatment, $F_{(1,28)}=$ $2.365, p=0.135$; DBS treatment, $F_{(1,28)}=1.095, p=0.304$; interac- 
A
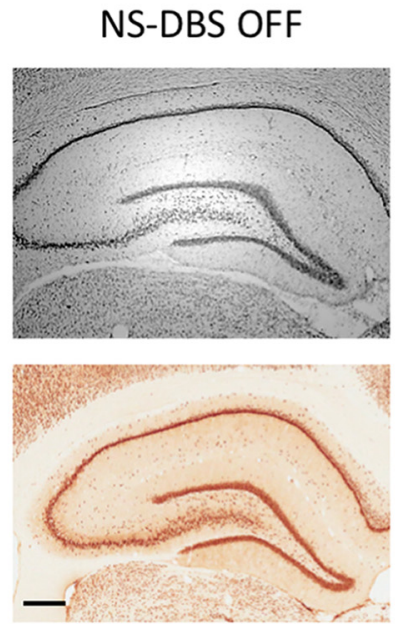

B

CA1

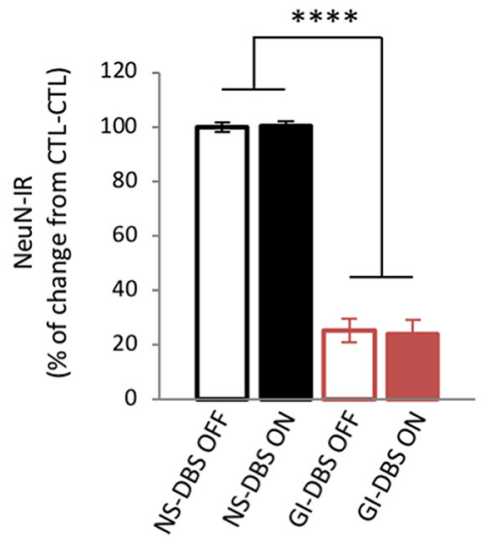

NS-DBS ON
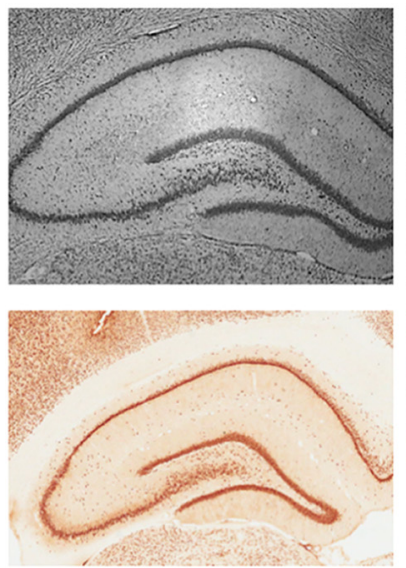

GI-DBS OFF
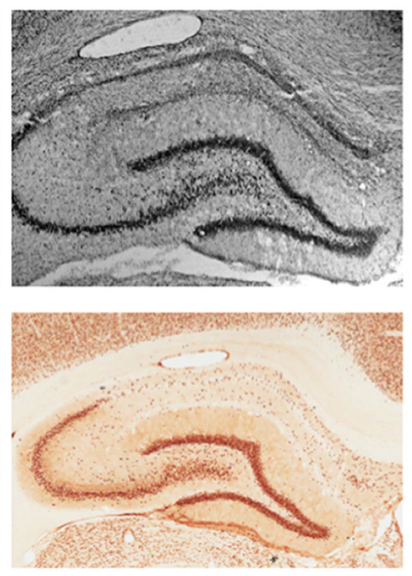

\section{GI-DBS ON}
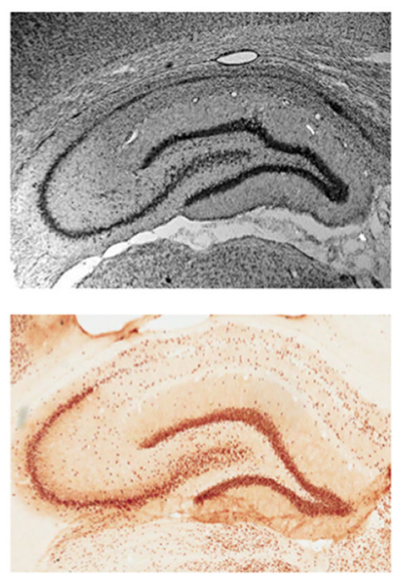

CA3

DG
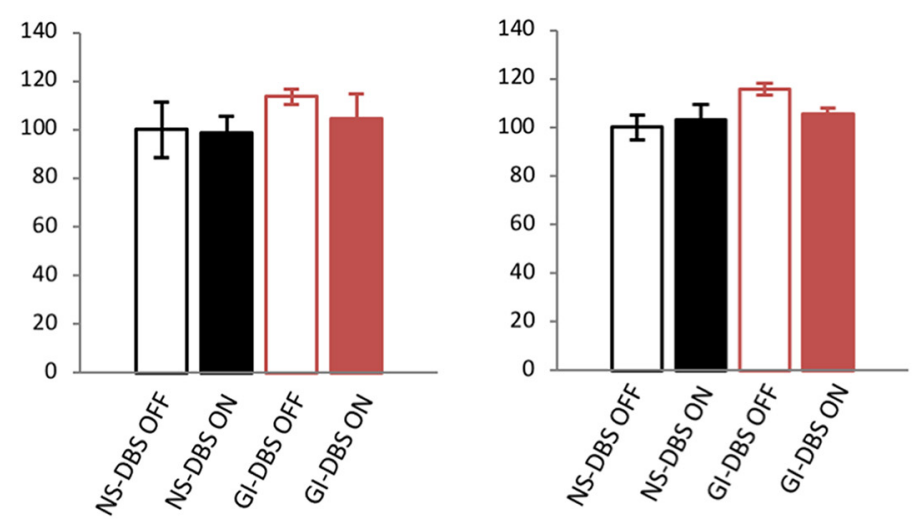

Figure 4. Histological characterization of hippocampal neurons following GI and DBS treatments. A, Representative photomicrographs of immunohistochemistry using Cresyl Violet (CV; top panels) staining and neuronal nuclear antigen (NeuN; bottom panels) staining in the hippocampus of the four different groups of animals. CV and NeuN staining showed a neuronal degeneration in the hippocampal Cornu Ammonis regions of CA1 for the groups of rats that received GI. Scale bar, $800 \mu \mathrm{m}$. B, Quantification of NeuN ${ }^{+}$cell in CA1 (left), CA3 (middle), and dentate gyrus (right). GI-DBS OFF and GI-DBS ON groups exhibited a significant decrease in NeuN ${ }^{+}$cells in CA1 compared with sham groups. The number of NeuN ${ }^{+}$cells remained unchanged in CA3 and dentate gyrus among the four groups. Two-way ANOVA followed by Tukey's post hoc test. ${ }^{* * *} p=0.0001 ; n=6 /$ group. Data are presented as the mean \pm SEM.

tion, $F_{(1,20)}=2.832, p=0.104$; Fig. $\left.6 B\right)$ among the four groups. These results show that DBS attenuates the loss of synaptophysin in CA1 induced by GI.

\section{DBS increased dendritic conductance after GI}

Electrical field potentials were elicited by stimulating the Schaffer collateral afferents at the stratum radiatum of the $\mathrm{CA} 2 / 3$ region using a bipolar stimulating electrode. In the CA1 somatic area [local field potential-somatic (LFP-som)], a typical population spike (PS) could be recorded in both NS-DBS OFF ( $n=5$ slices of $N=4$ animals) and NS-DBS ON ( $n=5$ of $N=5)$ groups, with average amplitudes of no significant difference $(3.39 \pm 0.2$ and $3.08 \pm 0.13 \mathrm{mV}$, respectively; $p>0.05$; Fig. $7 A, B)$. On the contrary, PS was never seen in animals after GI insult, regardless of DBS treatment or not $(n=8$ of $N=6$ GI-DBS OFF and $n=6$ of $N=5$ GI-DBS ON).

When recorded from the dendritic areas of the CA1 region (LFP-den), downward deflections could be induced in the four groups (Fig. 7C-F). These downward field potentials were clearly wider than the orthodromically evoked downward deflecting field potentials measured from the somatic area (Fig. 7A, classically interpreted as population spikes), compatible with their be- ing mainly field EPSPs (fEPSPs). Two-way ANOVA revealed no statistical differences in the slope (GI treatment, $F_{(1,16)}=2.34$, $p=0.146$; DBS treatment, $F_{(1,16)}=0.094, p=0.763$; interaction, $F_{(1,16)}=0.127, p=0.726$; Fig. $7 E$ ) but a significant main effect on the amplitudes of the downward deflection (LFP-den) in CA1 (GI treatment, $F_{(1,16)}=187.8, p<0.0001 ;$ DBS treatment, $F_{(1,16)}$ $=43.31, p<0.0001$; interaction, $F_{(1,16)}=9.29, p=0.008$; Fig. $7 D)$. No difference was found between NS-DBS OFF (1.21 \pm 0.09 $\mathrm{mV} ; n=4$ of $N=4)$ and NS-DBS ON $(1.43 \pm 0.07 \mathrm{mV} ; n=5$ of $N=5$ ) groups ( $p=0.122$; Fig. 7 ). The amplitudes of LFP-den recorded in GI-DBS OFF group $(n=6$ of $N=6)$ were dramatically smaller than the ones recorded in NS-DBS OFF $(0.20 \pm 0.03$ vs $1.21 \pm 0.09 \mathrm{mV}$, respectively; $p<0.0001)$ as well as NS-DBS $\mathrm{ON}$ rats $(0.20 \pm 0.03$ vs $1.43 \pm 0.07 \mathrm{mV}$, respectively; $p<$ 0.0001). Interestingly, although LFP-den amplitude of GI-DBS ON rats $(n=5$ of $N=5)$ was significantly smaller than the ones from NS-DBS OFF $(p=0.001)$ and NS-DBS ON $(p<0.001)$, fEPSP amplitudes differed markedly between the GI-DBS OFF group and the GI-DBS ON group. The GI-DBS ON group had a significantly larger amplitude of LFP-den than those recorded from GI-DBS OFF rats $(0.20 \pm 0.03$ vs $0.78 \pm 0.04 \mathrm{mV}$, respectively; $p<0.0001)$. Moreover, two-way ANOVA revealed a sig- 
A

DBS OFF
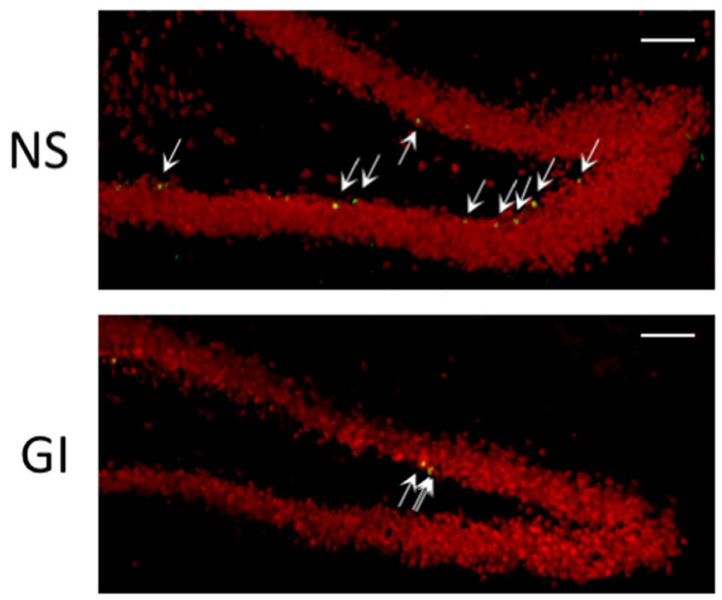

B

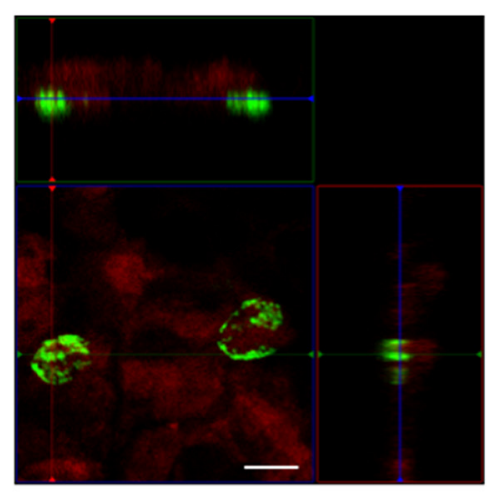

DBS ON
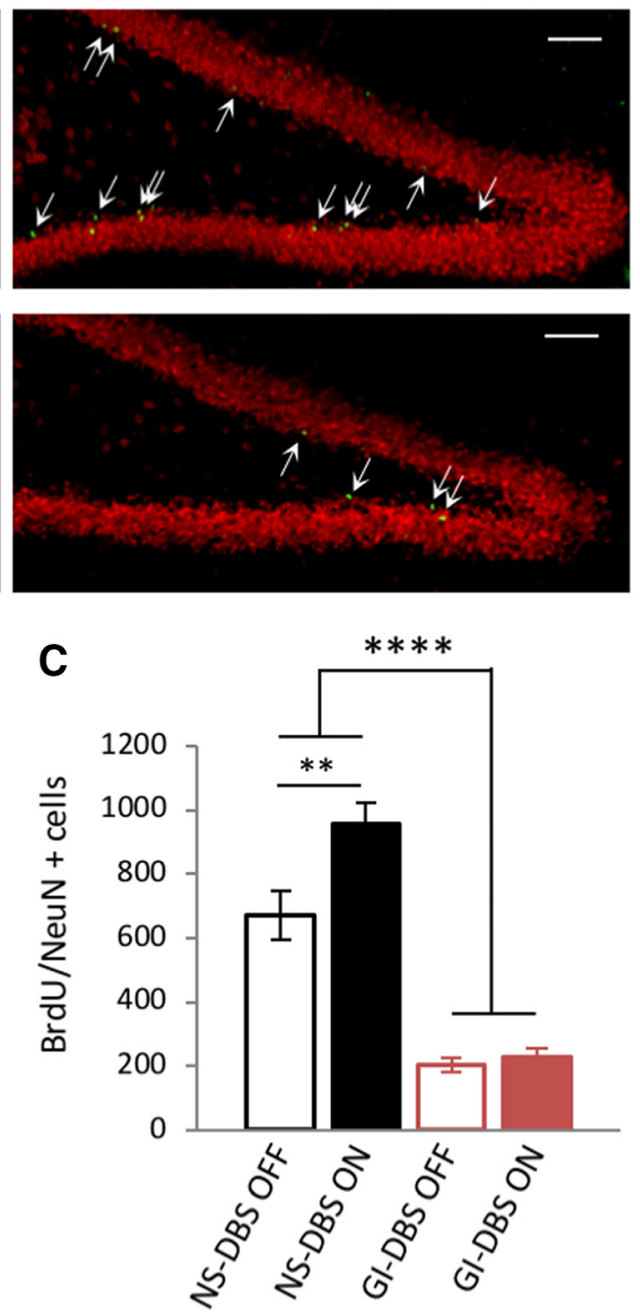

Figure 5. Gl induced a decrease in hippocampal neurogenesis. $A$, Representative photomicrographs of $\mathrm{BrdU}^{+}$(green) and $\mathrm{NeuN}^{+}$(red) immunofluorescence in the hippocampal subgranular zone of NS-DBS OFF, NS-DBS ON, GI-DBS OFF, and GI-DBS ON rats. Scale bar, $100 \mu \mathrm{m}$. B, Representative confocal orthogonal z-stack image, at high magnification, showing colocalization of BrdU ${ }^{+}$ (green) and $\mathrm{NeuN}^{+}$(red) cells. Scale bar, $10 \mu \mathrm{m}$. C, Quantification of immunoreactive BrdU ${ }^{+} / \mathrm{NeuN}^{+}$cells in the DG. Two-way ANOVA showed a significant main effect on the number of $\mathrm{BrdU}^{+} / \mathrm{NeuN}^{+}$cells. GI-DBS OFF and GI-DBS ON rats had significantly fewer BrdU ${ }^{+} / \mathrm{NeuN}^{+}$cells than both NS control groups $(p<0.001)$. Two-way ANOVA followed by Tukey's post hoc test. ${ }^{* *} p<0.01,{ }^{* * * *} p<0.0001 . n=8$ rats/group. Data are presented as the mean \pm SEM.

nificant main effect on the LFP-den amplitudes across stimulation intensities, as shown in the input/output $(\mathrm{I} / \mathrm{O})$ curves (DBS treatment, $F_{(1,9)}=116.7, p<0.0001$; amplitude, $F_{(4,36)}=$ $71.49, p<0.0001$; interaction, $\left.F_{(4,36)}=28.24, p<0.0001\right)$. The I/O curve of GI-DBS ON rats was significantly bigger than the one recorded in GI-DBS OFF slices across stimulation intensities $(p<0.0001$; Fig. $7 F)$. These results indicate that EC stimulation rescues certain aspects of the neuronal functional impairment induced by GI by enhancing the electrophysiological behavior at the dendritic site.

\section{Discussion}

Here we investigated the effect of EC DBS on spatial memory in a rat model of GI. We found that a single DBS treatment applied 2 weeks after GI induction is sufficient to rescue spatial memory deficits in GI rats trained 6 weeks after stimulation.

GI mimics the neurological consequences occurring after cardiac arrest by inducing neuronal death specifically in the hippocampal CA1 region (Petito et al., 1987; Schmidt-Kastner and
Freund, 1991), as illustrated by the dramatic decrease of $\mathrm{NeuN}^{+}$ cells in GI rats (Fig. 4). Selective CA1 lesions are well documented to impair hippocampal-dependent memory, such as spatial learning and memory performance in the MWM (Morris et al., 1982; Nunn et al., 1994; Block, 1999; Hartman et al., 2005). In line with these observations, we found that the dramatic loss of CA1 neurons in GI-DBS OFF rats was correlated with memory impairment in the MWM (Figs. 3, 4). Interestingly, despite the loss of CA1 neurons in GI-DBS ON rats, we found that they not only performed significantly better than GI-DBS OFF rats during a probing test, but memory performance was rescued to a level similar to those of NS control groups (Fig. 3B). These results are consistent with our previous findings showing that DBS improves memory performance in wild-type animals and animals with Alzheimer's-like pathology (Stone et al., 2011; Xia et al., 2017; Mann et al., 2018). Indeed, 6 weeks after receiving $1 \mathrm{~h}$ of EC DBS, naive C57BL/6 mice exhibited significant improvement in MWM performance compared with nonstimulated mice (Stone 
A

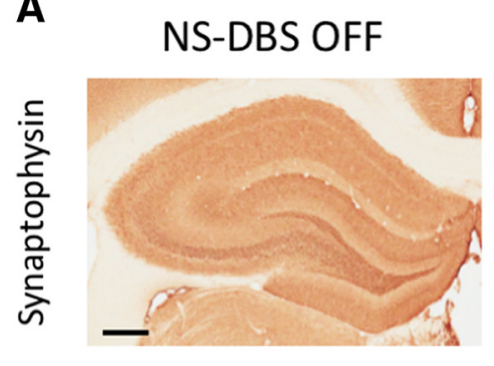

B

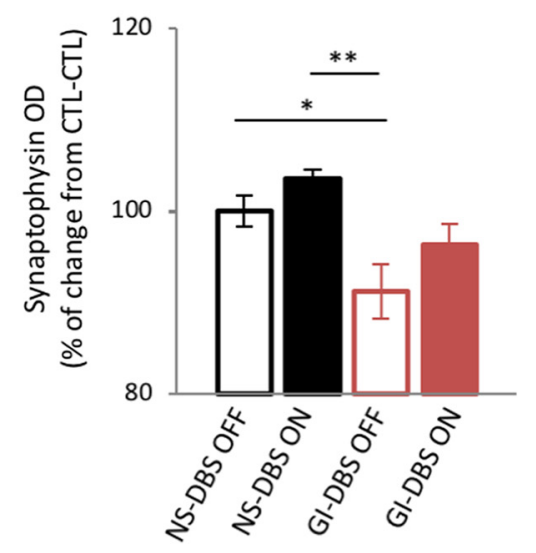

NS-DBS ON

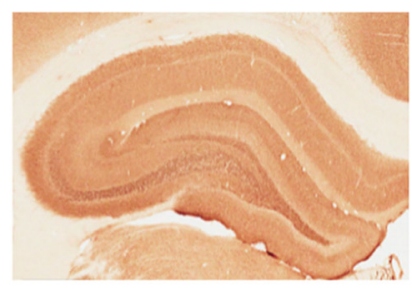

GI-DBS OFF

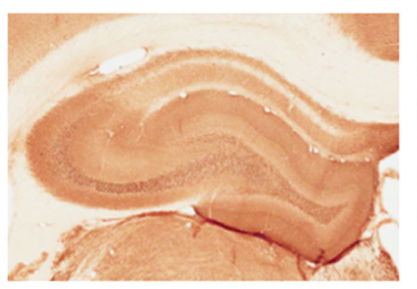

GI-DBS ON

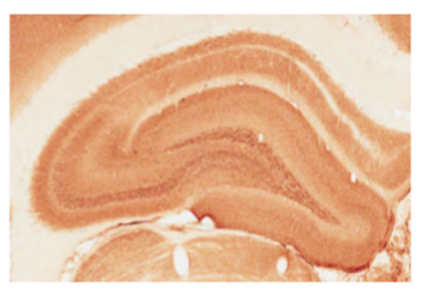

CA3

DG

Figure 6. DBS treatment attenuated the decrease in synaptophysin level in CA1 after $\mathrm{GI}$. $A$, Representative bright-field photomicrograph of synaptophysin immunoreactivity in the hippocampus of the four different groups of animals. Scale bar, $600 \mu \mathrm{m}$. B, Optic density measurement of synaptophysin expression in CA1 (left), CA3 (middle), and dentate gyrus (right). The level of synaptophysin in CA1 was significantly lower in GI-DBS OFF rats compared with NS-DBS OFF and NS-DBS ON rats. No significant differences were observed between GI-DBS ON and NS-DBS OFF, NS-DBS ON, and GI-DBS OFF. Synaptophysin levels remained unchanged in CA3 and dentate gyrus among the four groups. Two-way ANOVA followed by Tukey's posthoc test: ${ }^{*} p=0.05$, ${ }^{* *} p=0.01$. $n=8 /$ group. Data are presented as the mean \pm SEM.

et al., 2011). The beneficial effects of DBS in memory function may extend to other cognitive disorders. For example, Hao et al. (2015) reported that fornix DBS ameliorated memory deficits in a mouse model of Rett syndrome. Furthermore, we found that chronic EC DBS in a 3xTg AD mouse model improved spatial memory in the MWM as well as novel object recognition and novel object location performances, effects that outlast the stimulation period (Mann et al., 2018). Beneficial effects of DBS we described here and observed in these other studies persist at least 6 weeks after discontinuing stimulation, suggesting that DBS induces long-term changes in brain function. Several possible mechanisms can underlie these striking behavioral effects, such as DBS-induced neurogenesis in the DG, the rescue of CA1 neuronal death, and/or long-term plastic changes leading to improvements in synaptic efficiency and circuit activity.

While in some circumstances DBS-induced neurogenesis can contribute to memory performance enhancement in normal and disease models (Shors et al., 2001; Schinder and Gage, 2004; Deng et al., 2010; Stone et al., 2011), this does not appear to be a major mechanism in the context of ischemia, as shown here. We found that endogenous neurogenesis was dramatically reduced 2.5 weeks after GI induction and that EC DBS could not increase neurogenesis after GI induction (Fig. 5). The reasons for the apparent failure to induce neurogenesis in the context of the experiments we show in this article may be related to damage to progenitor cells related to the severity of the ischemic lesion or may be technical, including the nature and timing of BrdU injections or the brevity of the DBS stimulation duration, which lasted only $1 \mathrm{~h}$. Chronic stimulation may be better in inducing neuro- genesis, as shown in previous studies (Hao et al., 2015; Mann et al., 2018). Contrary to our previous findings in naive mice (Stone et al., 2011), we did not find cognitive changes within our NS groups, despite an increase in neurogenesis in the NS-DBS ON group. Previous studies (Frick et al., 2000; Stranahan, 2011) have reported differences between rats and mice when tested in the MWM; naive rats learned faster and performed better during the probe trial than naive mice. It is possible that we reached a floor effect of memory retention, and thus we could not detect small changes within our NS groups.

Another potential mechanism that may be involved in DBSimproved behavior are changes in synaptic density or plasticity. Consistent with other reports (Ishimaru et al., 2001; Sun et al., 2008), we found that GI led to a dramatic loss of CA1 neurons and a reduction of synaptophysin expression. This cell loss could not be rescued by DBS applied 2 weeks after an ischemic episode (Fig. 4). While DBS could not overcome the neuronal loss, DBS did, however, increase synaptophysin levels in CA1 after GI (Fig. 6). Although no significant differences were observed between GIDBS ON and GI-DBS OFF, the synaptophysin level in GI-DBS $\mathrm{ON}$ animals was increased to a level that was not significantly different from that in both NS groups, suggesting that DBS could partially restore CA1 synapse loss induced by GI. We reported previously that acute fornix DBS in rats rapidly increases hippocampal expression of synaptophysin and GAP-43, another synaptic plasticity marker (Gondard et al., 2015). Moreover, chronic EC DBS significantly enhanced the expression of CA1 synaptophysin in an $\mathrm{AD}$ mouse model compared with nonstimulated AD mice (Akwa et al., 2018). These results are supported by 
A

\section{NS-DBS OFF}

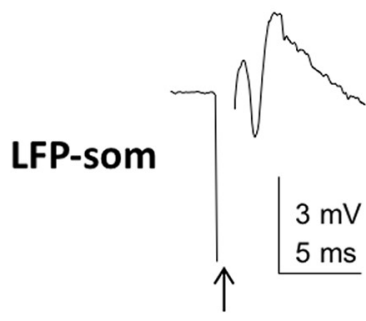

C

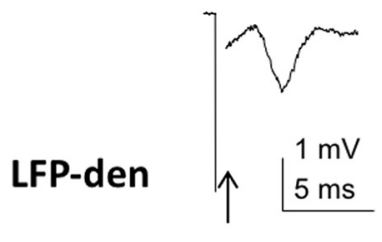

NS-DBS ON

\section{GI-DBS OFF}
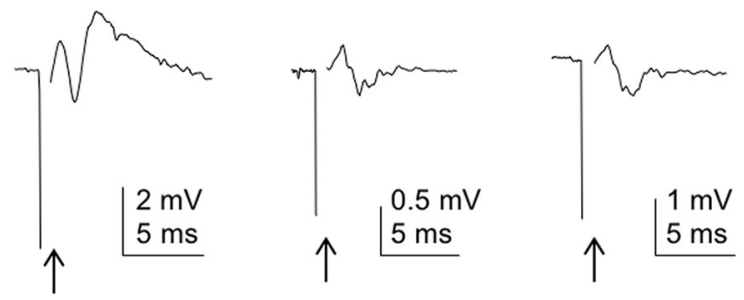

\section{GI-DBS ON}

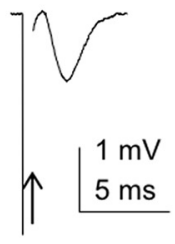

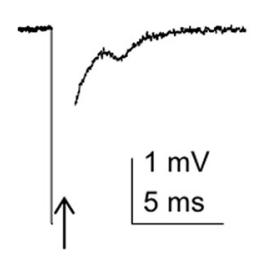

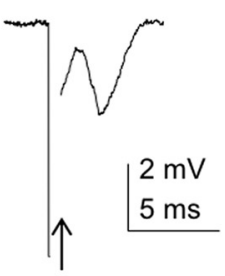

B
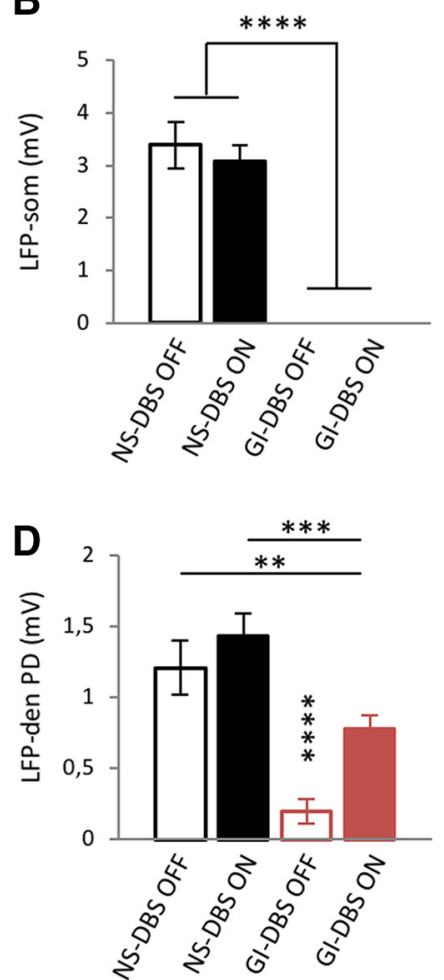

E

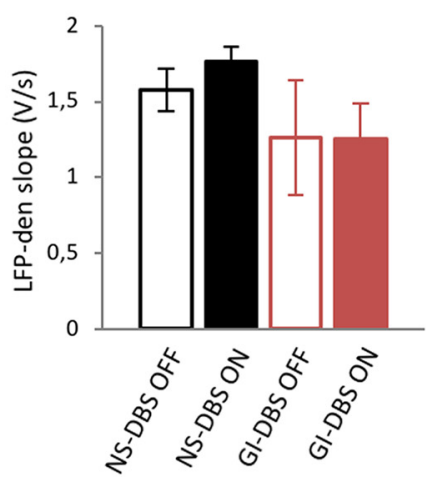

$\mathbf{F}$

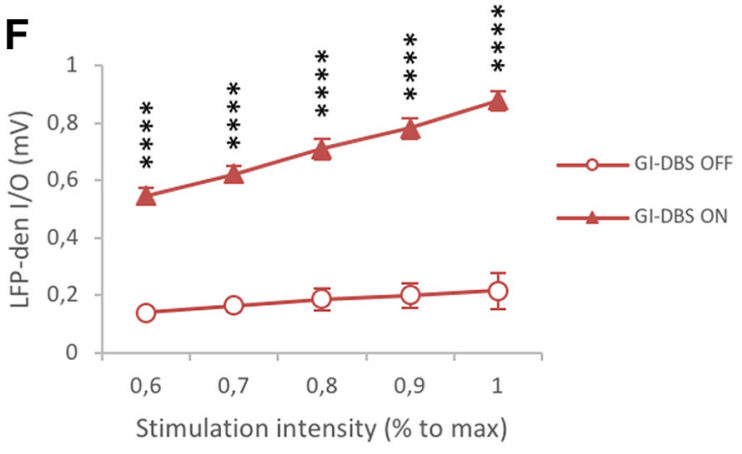

Figure 7. DBS treatment improved Gl-induced impaired evoked dendritic synaptic activity in the CA1 region. $A$, Representative PS recording in the CA1 somatic area (LFP-som) of the four different groups of animals. $B$, Average of LFP-som amplitudes (in $\mathrm{mV}$ ). No difference was observed in the two sham groups, and no LFP-som could be recorded in GI groups, regardless of the DBS treatment. $\boldsymbol{C}$, Representative local field potential recording from the dendritic areas (LFP-den) of the CA1 region of the four different groups of animals. $\boldsymbol{D}$, Average of LFP-den slope (in mV). $\boldsymbol{E}$, Average of LFP-den amplitudes (in mV). No difference was found between NS-DBS OFF ( $n=4$ of $N=4)$ and NS-DBSON $(n=5$ of $N=5)$ groups. The GI-DBS ON group ( $n=5$ of $N=5)$ had significantly larger amplitudes of LFP-den than the ones recorded from GI-DBS OFF rats $(n=6$ of $N=6)$. $F$, I/O curves obtained by plotting the slope of LFP-den of GI-DBS OFF and GI-DBS ON rats as a function of the stimulation intensity (from 0.6 to $1 \mathrm{~mA}$ ). Two-way ANOVA followed by Tukey's post hoc test: ${ }^{* *} p=0.01,{ }^{* * *} p=0.001 ;{ }^{* * *} p=0.0001$. Data are presented as the mean \pm SEM.

similar findings in a rat model of focal cortical ischemia, where chronic $30 \mathrm{~Hz}$ cerebellar stimulation induced improved locomotion deficits; enhanced the expression of synaptic markers of long-term potentiation (LTP) and plasticity, including synaptophysin, NMDAR1, CaMKII, and PSD-95; and increased the number of synapses (Machado et al., 2013; Cooperrider et al., 2014).

Although synaptophysin level indicates the likelihood of a higher number of synapses, its measurement does not allow distinction between active and inactive synapses. Given that synaptophysin was elevated in CA1, we performed in vitro electrophysiological recordings in this area. In addition to its effects on synaptic density, we found that DBS treatment could improve the electrophysiological properties of CA1 neurons in GI-DBS ON rats (Fig. 7). In agreement with our results, studies have reported alterations of electrophysiological properties following GI, such as changes in spontaneous activity accompanied with the decrease and eventual disappearance of the Schaffer collateral evoked responses in CA1 (Buzsàki et al., 1989; Hori and Carpenter, 1994). In fact, contrary to NS control groups, we could not detect a PS in animals that received GI, regardless of DBS treatment or not. These results indicate that GI largely affected synchronized neuronal firing activities within the CA1 region, either by reducing the actual number of neurons that could generate action spikes or the level of synchronization of local neurons. Moreover, LTP could not be induced in our GI groups, in agreement with other studies reporting alterations of LTP after ischemia (Kiprianova et al., 1999; Sun et al., 2009; Li et al., 2010). However, when recorded from CA1 dendritic areas, LFPs could be induced in all groups (Fig. 7C$F$ ). Studies showed that LFP from dendritic region contains both synaptic and nonsynaptic activities (Vizi and Lábos, 1991; Buzsáki et al., 2012). In this study, the evident field synaptic transmission did not differ among the four groups according to the slope of the rising phase of LFPs, which were not significantly different. However, consistent with the reported decrease in fEPSP amplitude following GI 
(Hori and Carpenter, 1994), we observed that LFP-den amplitude in the GI-DBS OFF group was dramatically smaller compared with both NS control groups. Despite the fact that DBS did not change the LFP-den amplitude in NS-DBS ON animals compared with NSDBS OFF rats, the amplitude of LPF-den in the GI-DBS ON group was significantly larger than the ones recorded from GI-DBS OFF rats. This change is not due to synchronized firing in the somatic region as no PSs were recorded in GI animals. Thus, the improvement of electrophysiological behavior is likely due to an increase in dendritic electrophysiological activity, which could be both synaptic and nonsynaptic. Although the slope of fEPSP is considered as the true measurement of synaptic transmission in the dendritic region, the fact that the small responses elicited in the GI-DBS OFF animals, to some extent, limited the comparability of the two GI groups. Therefore, changes in synaptic activities after DBS treatment cannot be ruled out through other nonsynaptic ongoing events might also contribute to the increase in amplitude of LFPs. These results are supported by previous studies in a rat model of cerebral ischemia, which shows DBS targeting the DTC pathway enhances cortical plasticity and excitability, and promotes perilesional cortical reorganization (Cooperrider et al., 2014; Park et al., 2015).

Previous studies investigated the role of neurotrophic factors in electrophysiolocal properties following GI. For example, BDNF administration could improve LTP induction that was impaired after GI (Kiprianova et al., 1999). Furthermore, intranasal administration of VEGF ameliorated cognitive function following GI and increased spontaneous EPSCs in the early stage of ischemia (Yang et al., 2014). We have previously shown that acute fornix DBS in rats modulate hippocampal expression of these two neurotrophic factors (Gondard et al., 2015), suggesting that they could participate in the increased synaptic connections to enhance the hippocampal function observed here; however, this remains to be tested.

Overall, our data from a rat model of GI support the notion that high-frequency EC DBS produces improvement in memory function. In this study, we used the DBS parameters currently used for treatment of movement disorders and under active investigation for the treatment of neuropsychiatric disorders (Lozano and Lipsman, 2013), including AD (Lozano et al., 2016). To our knowledge, however, DBS has never been examined for its cognitive benefits in the context of hypoxic-ischemic injuries. Our study recreates the clinical scenario in which stroke would be followed several days/weeks later by surgical implantation of electrodes. We found these beneficial effects may be mediated by the enhancement of synaptic plasticity and neuronal excitability, which are essential to hippocampal learning and memory. It is remarkable that DBS could exert such striking benefits to cognitive impairments caused by GI with a brief duration of stimulation $(1 \mathrm{~h})$. We have previously seen enhancement of neurogenesis and learning after similar acute DBS application (Stone et al., 2011). We speculate that chronic continuous stimulation, as used clinically, may lead to more potent changes in synaptic density and activity. The beneficial results of DBS on memory shown here are complemented by observations in other models of neurological disorders. We have previously shown that acute $(1 \mathrm{~h})$ and chronic EC DBS can restore memory functions in different mouse models of AD (Xia et al., 2017; Mann et al., 2018). These robust findings in $\mathrm{AD}$ mice support the promising effects of $\mathrm{DBS}$ in $\mathrm{AD}$ patients regarding cognitive function, disease progression, and increased cortical glucose metabolism (Laxton et al., 2010; Sankar et al., 2015). Together with accumulating evidence from $\mathrm{AD}$ and Rett syndrome animal models, and patients with $\mathrm{AD}$, the present study extends a possible role for DBS in the treatment of cognitive impairment associated with additional neurological disorders, including ischemia. The results of the present study allow for a better understanding of the action mechanism of DBS and represent a step toward DBS assessment as a potential therapy for ameliorating not only motor deficits (Elias et al., 2018) but, more particularly, memory and cognitive deficits associated with ischemia.

\section{References}

Adkins-Muir DL, Jones TA (2003) Cortical electrical stimulation combined with rehabilitative training: enhanced functional recovery and dendritic plasticity following focal cortical ischemia in rats. Neurol Res 25:780-788.

Akwa Y, Gondard E, Mann A, Capetillo-Zarate E, Alberdi E, Matute C, Marty S, Vaccari T, Lozano AM, Baulieu EE, Tampellini D (2018) Synaptic activity protects against $\mathrm{AD}$ and $\mathrm{FTD}$-like pathology via autophagiclysosomal degradation. Mol Psychiatry 23:1530-1540.

Azad TD, Veeravagu A, Steinberg GK (2016) Neurorestoration after stroke. Neurosurg Focus 40:E2.

Block F (1999) Global ischemia and behavioural deficits. Prog Neurobiol 58:279-295.

Buzsàki G, Freund TF, Bayardo F, Somogyi P (1989) Ischemia-induced changes in the electrical activity of the hippocampus. Exp Brain Res 78: $268-278$.

Buzsáki G, Anastassiou CA, Koch C (2012) The origin of extracellular fields and currents-EEG, ECoG, LFP and spikes. Nat Rev Neurosci 13:407420.

Colbourne F, Li H, Buchan AM, Clemens JA (1999) Continuing postischemic neuronal death in $\mathrm{CA} 1$ : influence of ischemia duration and cytoprotective doses of NBQX and SNX-111 in rats. Stroke 30:662-668.

Cooperrider J, Furmaga H, Plow E, Park HJ, Chen Z, Kidd G, Baker KB, Gale JT, Machado AG (2014) Chronic deep cerebellar stimulation promotes long-term potentiation, microstructural plasticity, and reorganization of perilesional cortical representation in a rodent model. J Neurosci 34: $9040-9050$

Deng W, Aimone JB, Gage FH (2010) New neurons and new memories: how does adult hippocampal neurogenesis affect learning and memory? Nat Rev Neurosci 11:339-350.

Desmond DW, Moroney JT, Paik MC, Sano M, Mohr JP, Aboumatar S, Tseng CL, Chan S, Williams JB, Remien RH, Hauser WA, Stern Y (2000) Frequency and clinical determinants of dementia after ischemic stroke. Neurology 54:1124-1131.

Elias GJB, Namasivayam AA, Lozano AM (2018) Deep brain stimulation for stroke: current uses and future directions. Brain Stimul 11:3-28.

Encinas JM, Hamani C, Lozano AM, Enikolopov G (2011) Neurogenic hippocampal targets of deep brain stimulation. J Comp Neurol 519:6-20.

Frick KM, Stillner ET, Berger-Sweeney J (2000) Mice are not little rats: species differences in a one-day water maze task. Neuroreport 11:3461-3465.

Gondard E, Chau HN, Mann A, Tierney TS, Hamani C, Kalia SK, Lozano AM (2015) Rapid modulation of protein expression in the rat hippocampus following deep brain stimulation of the fornix. Brain Stimul 8:1058-1064.

Hamani C, McAndrews MP, Cohn M, Oh M, Zumsteg D, Shapiro CM, Wennberg RA, Lozano AM (2008) Memory enhancement induced by hypothalamic/fornix deep brain stimulation. Ann Neurol 63:119-123.

Hamani C, Stone SS, Garten A, Lozano AM, Winocur G (2011) Memory rescue and enhanced neurogenesis following electrical stimulation of the anterior thalamus in rats treated with corticosterone. Exp Neurol 232: $100-104$.

Hao S, Tang B, Wu Z, Ure K, Sun Y, Tao H, Gao Y, Patel AJ, Curry DJ, Samaco RC, Zoghbi HY, Tang J (2015) Forniceal deep brain stimulation rescues hippocampal memory in rett syndrome mice. Nature 526:430-434.

Hartman RE, Lee JM, Zipfel GJ, Wozniak DF (2005) Characterizing learning deficits and hippocampal neuron loss following transient global cerebral ischemia in rats. Brain Res 1043:48-56.

Hescham S, Lim LW, Jahanshahi A, Steinbusch HW, Prickaerts J, Blokland A, Temel Y (2013) Deep brain stimulation of the forniceal area enhances memory functions in experimental dementia: the role of stimulation parameters. Brain Stimul 6:72-77.

Hori N, Carpenter DO (1994) Functional and morphological changes induced by transient in vivo ischemia. Exp Neurol 129:279-289.

Ishimaru H, Casamenti F, Uéda K, Maruyama Y, Pepeu G (2001) Changes in presynaptic proteins, SNAP-25 and synaptophysin, in the hippocampal CA1 area in ischemic gerbils. Brain Res 903:94-101. 
Kalia SK, Sankar T, Lozano AM (2013) Deep brain stimulation for Parkinson's disease and other movement disorders. Curr Opin Neurol 26:374-380.

Kiprianova I, Sandkühler J, Schwab S, Hoyer S, Spranger M (1999) Brainderived neurotrophic factor improves long-term potentiation and cognitive functions after transient forebrain ischemia in the rat. Exp Neurol 159:511-519.

Lackland DT, Roccella EJ, Deutsch AF, Fornage M, George MG, Howard G, Kissela BM, Kittner SJ, Lichtman JH, Lisabeth LD, Schwamm LH, Smith EE, Towfighi A (2014) Factors influencing the decline in stroke mortality: a statement from the American Heart Association/American Stroke Association. Stroke 45:315-353.

Laxton AW, Tang-Wai DF, McAndrews MP, Zumsteg D, Wennberg R, Keren R, Wherrett J, Naglie G, Hamani C, Smith GS, Lozano AM (2010) A phase I trial of deep brain stimulation of memory circuits in Alzheimer's disease. Ann Neurol 68:521-534.

Li S, He Z, Guo L, Huang L, Wang J, He W (2010) Behavioral alterations associated with a down regulation of HCN1 mRNA in hippocampal cornus ammon 1 region and neocortex after chronic incomplete global cerebral ischemia in rats. Neuroscience 165:654-661.

Lipsman N, Woodside DB, Giacobbe P, Hamani C, Carter JC, Norwood SJ, Sutandar K, Staab R, Elias G, Lyman CH, Smith GS, Lozano AM (2013) Subcallosal cingulate deep brain stimulation for treatment-refractory anorexia nervosa: a phase 1 pilot trial. Lancet 381:1361-1370.

Lozano AM, Fosdick L, Chakravarty MM, Leoutsakos JM, Munro C, Oh E, Drake KE, Lyman CH, Rosenberg PB, Anderson WS, Tang-Wai DF, Pendergrass JC, Salloway S, Asaad WF, Ponce FA, Burke A, Sabbagh M, Wolk DA, Baltuch G, Okun MS, et al. (2016) A phase II study of fornix deep brain stimulation in mild Alzheimer's disease. J Alzheimers Dis 54:777-787.

Lozano AM, Hamani C (2004) The future of deep brain stimulation. J Clin Neurophysiol 21:68-69.

Lozano AM, Lipsman N (2013) Probing and regulating dysfunctional circuits using deep brain stimulation. Neuron 77:406-424.

Machado AG, Cooperrider J, Furmaga HT, Baker KB, Park HJ, Chen Z, Gale JT (2013) Chronic 30-Hz deep cerebellar stimulation coupled with training enhances post-ischemia motor recovery and peri-infarct synaptophysin expression in rodents. Neurosurgery 73:344-353.

Madl C, Holzer M (2004) Brain function after resuscitation from cardiac arrest. Curr Opin Crit Care 10:213-217.

Mann A, Gondard E, Tampellini D, Milsted JAT, Marillac D, Hamani C, Kalia SK, Lozano AM (2018) Chronic deep brain stimulation in an Alzheimer's disease mouse model enhances memory and reduces pathological hallmarks. Brain Stimul 11:435-444.

Mayberg HS, Lozano AM, Voon V, McNeely HE, Seminowicz D, Hamani C, Schwalb JM, Kennedy SH (2005) Deep brain stimulation for treatmentresistant depression. Neuron 45:651-660.

Morris RG, Garrud P, Rawlins JN, O'Keefe J (1982) Place navigation impaired in rats with hippocampal lesions. Nature 297:681-683.

Mozaffarian D, Benjamin EJ, Go AS, Arnett DK, Blaha MJ, Cushman M, de Ferranti S, Després JP, Fullerton HJ, Howard VJ, Huffman MD, Judd SE, Kissela BM, Lackland DT, Lichtman JH, Lisabeth LD, Liu S, Mackey RH, Matchar DB, McGuire DK, et al. (2015) Heart disease and stroke statistics-2015 update: a report from the American Heart Association. Circulation 131:e29-e322.

Nunn JA, LePeillet E, Netto CA, Hodges H, Gray JA, Meldrum BS (1994) Global ischaemia: hippocampal pathology and spatial deficits in the water maze. Behav Brain Res 62:41-54.

Park HJ, Furmaga H, Cooperrider J, Gale JT, Baker KB, Machado AG (2015) Modulation of cortical motor evoked potential after stroke during electrical stimulation of the lateral cerebellar nucleus. Brain Stimul 8:1043-1048.
Paxinos G, Watson C (2008) The rat brain in stereotaxic coordinates, Ed 7. Amsterdam: Elsevier Academic.

Petito CK, Feldmann E, Pulsinelli WA, Plum F (1987) Delayed hippocampal damage in humans following cardiorespiratory arrest. Neurology 37:1281-1286.

Ponce FA, Lozano AM (2010) Deep brain stimulation state of the art and novel stimulation targets. Prog Brain Res 184:311-324.

Pulsinelli WA, Buchan AM (1988) The four-vessel occlusion rat model: method for complete occlusion of vertebral arteries and control of collateral circulation. Stroke 19:913-914.

Pulsinelli WA, Brierley JB, Plum F (1982) Temporal profile of neuronal damage in a model of transient forebrain ischemia. Ann Neurol 11:491-498.

Sankar T, Chakravarty MM, Bescos A, Lara M, Obuchi T, Laxton AW, McAndrews MP, Tang-Wai DF, Workman CI, Smith GS, Lozano AM (2015) Deep brain stimulation influences brain structure in Alzheimer's disease. Brain Stimul 8:645-654.

Schinder AF, Gage FH (2004) A hypothesis about the role of adult neurogenesis in hippocampal function. Physiology (Bethesda) 19:253-261.

Schmidt-Kastner R, Freund TF (1991) Selective vulnerability of the hippocampus in brain ischemia. Neuroscience 40:599-636.

Schweizer TA, Macdonald L (2014) The behavioral consequences of stroke (Schweizer TA, Macdonald L, eds). New York: Springer.

Shors TJ, Miesegaes G, Beylin A, Zhao M, Rydel T, Gould E (2001) Neurogenesis in the adult is involved in the formation of trace memories. Nature 410:372-376.

Smith GS, Laxton AW, Tang-Wai DF, McAndrews MP, Diaconescu AO, Workman CI, Lozano AM (2012) Increased cerebral metabolism after 1 year of deep brain stimulation in Alzheimer disease. Arch Neurol 69:1141-1148.

Stone SS, Teixeira CM, Devito LM, Zaslavsky K, Josselyn SA, Lozano AM, Frankland PW (2011) Stimulation of entorhinal cortex promotes adult neurogenesis and facilitates spatial memory. J Neurosci 31:13469-13484.

Stranahan AM (2011) Similarities and differences in spatial learning and object recognition between young male C57BL/6J mice and SpragueDawley rats. Behav Neurosci 125:791-795.

Sun HS, Jackson MF, Martin LJ, Jansen K, Teves L, Cui H, Kiyonaka S, Mori Y, Jones M, Forder JP, Golde TE, Orser BA, Macdonald JF, Tymianski M (2009) Suppression of hippocampal TRPM7 protein prevents delayed neuronal death in brain ischemia. Nat Neurosci 12:1300-1307.

Sun MK, Hongpaisan J, Nelson TJ, Alkon DL (2008) Poststroke neuronal rescue and synaptogenesis mediated in vivo by protein kinase $\mathrm{C}$ in adult brains. Proc Natl Acad Sci U S A 105:13620-13625.

Toda H, Hamani C, Fawcett AP, Hutchison WD, Lozano AM (2008) The regulation of adult rodent hippocampal neurogenesis by deep brain stimulation. J Neurosurg 108:132-138.

Vizi ES, Lábos E (1991) Non-synaptic interactions at presynaptic level. Prog Neurobiol 37:145-163.

Volpe BT, Petito CK (1985) Dementia with bilateral medial temporal lobe ischemia. Neurology 35:1793-1797.

von Euler M, Bendel O, Bueters T, Sandin J, von Euler G (2006) Profound but transient deficits in learning and memory after global ischemia using a novel water maze test. Behav Brain Res 166:204-210.

Xia F, Yiu A, Stone SSD, Oh S, Lozano AM, Josselyn SA, Frankland PW (2017) Entorhinal cortical deep brain stimulation rescues memory deficits in both young and old mice genetically engineered to model Alzheimer's disease. Neuropsychopharmacology 42:2493-2503.

Yang J, Yao Y, Chen T, Zhang T (2014) VEGF ameliorates cognitive impairment in in vivo and in vitro ischemia via improving neuronal viability and function. Neuromolecular Med 16:376-388. 